\title{
Experimental and Numerical Investigations on Crack Propagation in Titanium Alloys
}

\author{
Jyothirmayi Narne, Varimadugu Sandhya*, Nagini Yerramsetty and V. Jaipal Reddy \\ Department of Mechanical Engineering, Chaitanya Bharathi Instiute of Technology, Gandipet, Hyderabad-500075, Telangana State, India \\ Received 01 May 2018, Accepted 01 July 2018, Available online 02 July 2018, Vol.8, No.4 (July/Aug 2018)
}

\begin{abstract}
The titanium alloys are the objects of wide experimental analysis in the terms of crack growth characteristics and mechanism of fracture due to their applications. The Ti-6.4\%Al-2.6\%Mo-1.7\%Cr-0.5\%Fe-0.5\%Si (wt \%) alloy was used in the investigation. The tensile tests were conducted on plate specimens. The test variables considered are width of tensile specimen, crack size and tensile loading. The mechanism of fracture that appeared in the specimens was established from the TEM micrographs of the replicas taken from different zones of the fracture surfaces. The mechanism of fracture was also studied using finite element analysis. The results obtained from the FEA were verified with experimental results. It can be concluded that there is a general trend of increasing stress intensity factor with increasing applied tensile load, crack size and width of the flat specimen. The strain energy release increases with increasing applied tensile load. Crack extension can occur when crack-driving force is equal to the energy required for crack growth. For a particular stress the energy release rate is proportional to the crack size. As the tensile load and the size of the initial crack increase, there is an increased crack growth in the Ti alloy. In the crack initiation zone evidence of quasi-cleavage fracture with limited plastic striations symptoms was found in the specimen tested under $\sigma=1000 \mathrm{MPa}$. Micro fracture analysis of the specimens (tested under $\sigma=1100 \mathrm{MPa}$ ) has revealed quasi-cleavage fracture with small symptoms of plastic shearing in the early part of cracking.
\end{abstract}

Keywords: TEM micrographs, quasi-cleavage fracture, stress intensity factor, strain energy release, plastic shearing.

\section{Introduction}

Titanium alloys are widely used for a variety of applications such as military aircraft, atomic energy and ocean components of structures due to their high strength, good thermal stability and excellent corrosion resistance. Simultaneously, the titanium alloys possesses a lot of different structures resulted from manufacturing process, heat treatment and that the fracture properties may also change. Large varieties of micro structural features observed in titanium alloys such as grain sizes, globular or lamellar type of structures, $\beta$-phase or $(\alpha+\beta)$ phase morphologies as well as bimodal structures produce different resistance to crack initiation and crack propagation of fracture. For this reason the titanium alloys are the objects of wide experimental analysis in the terms of crack growth characteristics and mechanisms of fracture.

Sergio Baragetti (2014) studied the effect of stress concentration factor and the role of different inert or corrosive environments. He found the threshold stress intensity factor after which the environment has no

*Corresponding author's ORCID ID: 0000-0003-3136-0888 DOI: https://doi.org/10.14741/ijcet/v.8.4.5 effect on the fatigue damage. Kuntimaddi Sadananda et al., (2009) examined the role of stress range and the maximum stress in the fatigue crack nucleation and propagation. He also related this to both the stress life and fracture mechanics. Yang Cao et al., (2015) used experimental - numerical hybrid method to simulate the fracture behavior of MIL composite. Chenchong Wang et al., (2015) developed finite element model to study crack path and crack growth rate. Contour integral method was used to study the best thickness of austenite layer, the effect of crack propagation direction.

In this work, attention is paid on the Ti-6Al-3Mo$2 \mathrm{Cr}$ titanium alloy that finds applications as components of the aircraft engine. Analysis of crack growth in engine compressor disks made of this material was the subject of the work.

\section{Methodology}

The objectives of this work are:

- To study the fracture analysis of pre-cracked plates made of $\mathrm{Ti}-6 \mathrm{Al}-3 \mathrm{Mo}-2 \mathrm{Cr}$ titanium alloy experimentally. 
- To study the fracture behaviour of pre-cracked plates using Finite Element Analysis

The Ti-6.4\%Al-2.6\%Mo-1.7\%Cr-0.5\%Fe-0.5\%Si (wt \%) alloy was used in the investigation. The material was subjected to heat treatment consisting of $900^{\circ} \mathrm{C}$ for one hour, furnace quenching to $650^{\circ} \mathrm{C}$, held at $650^{\circ} \mathrm{C}$ for two hours and then air cooled. Mechanical properties of the material were: ultimate tensile stress $\sigma_{\mathrm{u}}=968$ $1100 \mathrm{MPa}$, elongation $\mathrm{e}_{1}=15-20 \%$, reduction area $r_{a}=36-45 \%$. The structure of $(\alpha+\beta)$ type consisted of $\alpha-$ platelets $(90 \%)$ contained in large prior $\beta$-phase. The mean grain size of $\alpha$-grains was $6-8 \mu \mathrm{m}$.

The tensile tests were conducted on plate specimens. The test variables are width of tensile specimen, crack size and tensile loading. The mechanism of fracture that appeared in the specimens was established from the TEM micrographs of the replicas taken from different zones of fracture surfaces. The mechanism of fracture was also studied using Finite Element Analysis. The results obtained from the FEA were verified with experimental results.

\section{Finite Element Analysis}

Solving a fracture mechanics problem involves performing a linear elastic or elastic-plastic static analysis and then using specialized post-processing commands or macros to calculate desired fracture parameters. The two main aspects of this procedure are: Modeling the crack region and calculating fracture parameters.

\subsection{Modeling the crack region}

The most important region in a fracture model is the region around the edge of the crack. The edge of the crack is referred as crack tip in 2-D model and crack front in a 3-D model. In elastic problems, the displacements near the crack tip (or crack front) vary as $\sqrt{r}$, where $r$ is the distance from the crack tip. The stresses and strains are singular at the crack tip varying as $\frac{1}{\sqrt{r}}$. To pick up the singularity in the strain, the crack faces should be coincident and the elements around the crack tip(or crack front) should be quadratic, with the mid side nodes placed at the quarter points. Such elements are called singular elements.

The recommended element type for a twodimensional fracture model is Plane-82, the six-node triangular solid. The first row of elements around the crack tip should be singular. The PREP7 KSCON command (GUI path main menu>Preprocessor $>$ meshing-shape \& size $>$ concentrate KPs $>$ Create), which assigns element division sizes around a key point, is particularly useful in a fracture model. It automatically generates singular elements around the specified key point. Other fields on the command allow controlling the radius of the first row elements, number of elements in the circumferential direction etc. For reasonable results, the first row elements around the crack tip should have a radius of approximately a/8 or smaller, where a is the crack length. In the circumferential direction, roughly one element every 30 to 40 degrees is recommended. The crack tip elements should not be distorted, and should take the shape of the isosceles triangles.

The discretization of tensile test specimen is shown in Figure 1. The element chosen is PLANE-82. The analysis was assumed under plane strain conditions. 12-test conditions were modeled in this work (Table 1) and static analysis was done for each load case. The type of loading is tensile. The material properties are: elastic modulus $=96 \mathrm{GPa}$ and Poisson ratio $=0.3$

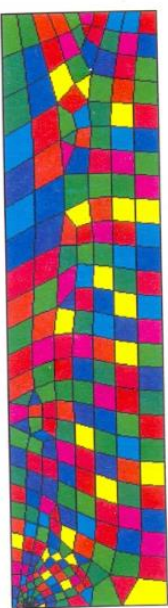

Fig. 1 Mesh of the tensile test specimen

Table 1 Test conditions

\begin{tabular}{|c|c|c|c|c|}
\hline $\begin{array}{c}\text { Test } \\
\text { No. }\end{array}$ & $\begin{array}{c}\text { Width of } \\
\text { specimen } \\
(\mathrm{mm})\end{array}$ & $\begin{array}{c}\text { Length of } \\
\text { specimen } \\
(\mathrm{mm})\end{array}$ & $\begin{array}{c}\text { Crack } \\
\text { Size } \\
(\mathrm{mm})\end{array}$ & $\begin{array}{c}\text { Loading } \\
(\mathrm{MPa})\end{array}$ \\
\hline 1 & 25 & 100 & 2.5 & 900 \\
\hline 2 & 25 & 100 & 2.5 & 1000 \\
\hline 3 & 25 & 100 & 2.5 & 1100 \\
\hline 4 & 50 & 100 & 2.5 & 900 \\
\hline 5 & 50 & 100 & 2.5 & 1000 \\
\hline 6 & 50 & 100 & 2.5 & 1100 \\
\hline 7 & 25 & 100 & 5.0 & 900 \\
\hline 8 & 25 & 100 & 5.0 & 1000 \\
\hline 9 & 25 & 100 & 5.0 & 1100 \\
\hline 10 & 50 & 100 & 5.0 & 900 \\
\hline 11 & 50 & 100 & 5.0 & 1000 \\
\hline 12 & 50 & 100 & 5.0 & 1100 \\
\hline
\end{tabular}

\subsection{Calculating Fracture Parameters}

Once the static analysis is completed, POST1, the general post processor is used to calculate fracture parameters. As mentioned earlier, typical fracture parameters of interest are stress intensity factors and the energy release rate.

\subsubsection{Stress Intensity Factors}

The POST1 KCALC command (GUI Path Main Menu $>$ General $>$ Postprocessor $>$ Nodal calculations > Stress Intensity Factors) calculates the mixed-mode 
stress intensity factors $\mathrm{K}_{\mathrm{I}}$. This command is limited to linear elastic problems with a homogeneous, isotropic material near the crack region. To use KCALC properly, following steps in POST1 should be followed:

Define a local Crack-tip or crack-front coordinate system, with X parallel to the crack face (perpendicular to the crack front in 3-D models) and Y perpendicular to the crack face.

Define a path along the crack face. The first node on the path should be the crack-tip node. For a half-crack model, two additional nodes are required, both along the crack face. For full-crack model, where both crack faces are included, four additional nodes are requiredtwo along one crack face and two along the other

Calculate $\mathrm{K}_{\mathrm{I}}$. The KPLAN field on the KCALC command specifies whether is the model is plane strain or plane stress. Except for the analysis of thin plates, the asymptotic or near-crack-tip behavior of stress is usually thought to be that of plane strain. The KCSYM field specifies whether the model is a half-crack model with symmetry boundary conditions or a full-crack model.

\subsubsection{Energy Release Rate}

Energy release is a concept used to determine the amount of work (change of energy) associated with a crack opening or closure. One method to calculate the energy release rate is the virtual crack extension method.

In the virtual crack extension method, two analyses are performed, one with crack length a and the other with crack length $a+\Delta a$. If the potential energy $U$ (strain energy) for both cases is stored, the energy release rate can be calculated from

$G=\frac{-U_{a+\Delta a}-U_{a}}{B \Delta a}$

Where B is the thickness of the fracture model.

The crack length is extended by $\Delta \mathrm{a}$ for the second analysis by selecting all nodes in the vicinity of the crack and scaling them in the $\mathrm{X}$ direction [NSCALE] (GUI path Main Menu> Preprocessor>-ModelingOperate-Scale) by the factor $\Delta$ a.

\section{Results and Discussion}

In this chapter the influence of specimen size, crack size and load variation on the stress intensity factor and strain release energy of Ti alloy is presented. The results obtained from the finite element analysis are verified with experimental results.

\subsection{Stress Intensity Factor}

Fracture-resistant design has two significant components. The first, which considers the applied stress state and the likelihood of flaws being present in the structure, allows for estimating service stresses in terms of flaw size and the material property, $\mathrm{K}_{\mathrm{I}}$. The influence of tensile load applied onto the specimen on stress intensity factor is shown in Figure 2. It can be seen a general trend of increasing stress intensity factor with increasing applied tensile load. It can also be observed that the stress intensity factor increases with increasing crack size and width of the flat specimen. Ideally, the curves should go zero stress intensity for zero applied loads. In this Figure, it can be seen that they do not; they do indicate some value of negative stress intensity, which is interpreted as a residual stress state that forces the crack surfaces together.

It is also revealed that the specimen having width = $50 \mathrm{~mm}$ and crack size of $5 \mathrm{~mm}$ exhibits steep rate of increasing stress intensity factor. The graph curvature is a polynomial of degree three. Under this condition, the crack growth is nonlinear and faster.

The stress intensity factors for 12 test conditions are obtained. The plot for one test condition is shown in Figure 3 . From all the graphs, it is understood that the stress intensity factor is very high at the crack tip. The crack growth increases with increase of tensile load and the initial crack size in the test specimen.

\subsection{Strain Release Energy}

The influence of tensile load on the strain energy is shown in Figure 4. Under all the test conditions, the strain energy increases with increasing applied tensile load. Crack extension can occur when crack-driving force is equal to the energy required for crack growth The energy required for a crack to grow in a metal is much larger than the surface energy to create the new free surfaces. In metals plastic deformation occurs in front of the crack and during crack extension energy is expended by the formation of a new plastic zone at the tip of the advanced crack. The fracture criterion in Ti alloy is depicted graphically in the way shown in Figure 4. For a particular stress the energy release rate is proportional to the crack size. As the tensile load and the size of the initial increase, there is an increased crack growth in the Ti alloy.

The strain release rates in the $\mathrm{Ti}$ alloy under one test condition is shown in the Figure. 5. It is observed that the energy release rate is maximum at the crack tip. This indicates that the crack opening is accelerated with increase of tensile load and initial crack size. The crack growth is very high in a tensile specimen having the conditions of width of plate $=50 \mathrm{~mm}$, crack size $=$ $5 \mathrm{~mm}$ and tensile load of $1100 \mathrm{MPa}$.

\section{Experimental Analysis of Fracture Mechanism}

The microstructures of fracture in the Ti alloy are shown in Figures 6 to 11. Mechanism of fracture that appeared in the specimens was established from the TEM micrographs of the replicas taken from different zones of the fracture surfaces. 
In the examined $\mathrm{Ti}$ alloy under tension the cracks were observed to be initiated at the interface between $\alpha$ and $\beta$ phases (Figure 6) and in $\alpha$-grains (Figure 7). The images were taken from the specimens tested under $\sigma=900 \mathrm{MPa}$.

In the crack initiation zone evidence of quasicleavage fracture with limited plastic striations symptoms was found in the specimen tested under $\sigma=$ 1000MPa (Figure 8). Plastic striations were occupied almost whole fracture surface of specimens (Figure 9).

Micro fracture analysis of the specimens (tested under $\sigma=1100 \mathrm{MPa}$ ) has revealed quasi-cleavage fracture with small symptoms of plastic shearing in the early part of cracking( Figure 10). It was found an interesting phenomenon that below certain depth the cleavage fracture was passed to the plastic striations. The striations were occupied greater part or almost whole fracture surface (Figure 11).

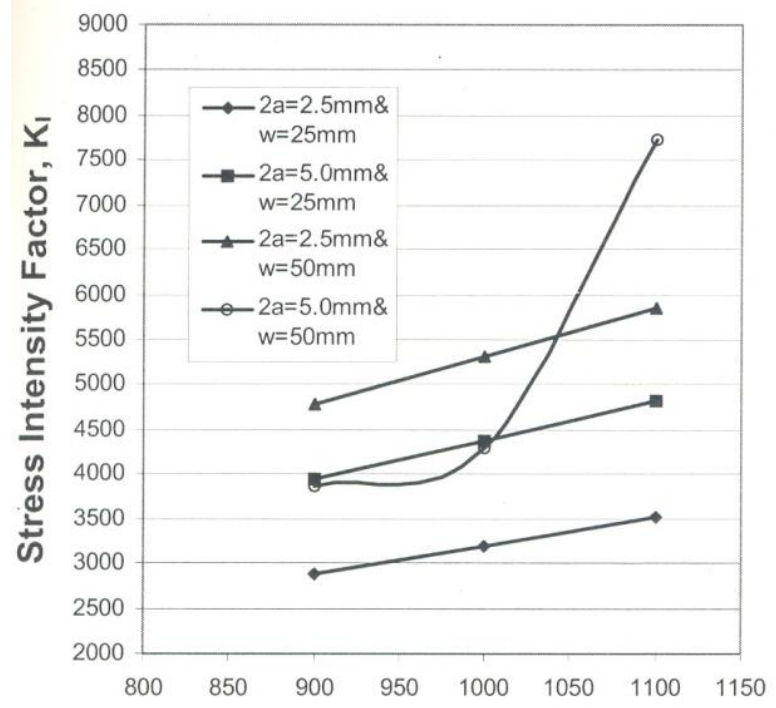

Applied tensile load, MPa/Sq.mm

Fig. 2 Variation of stress intensity factor with the applied load

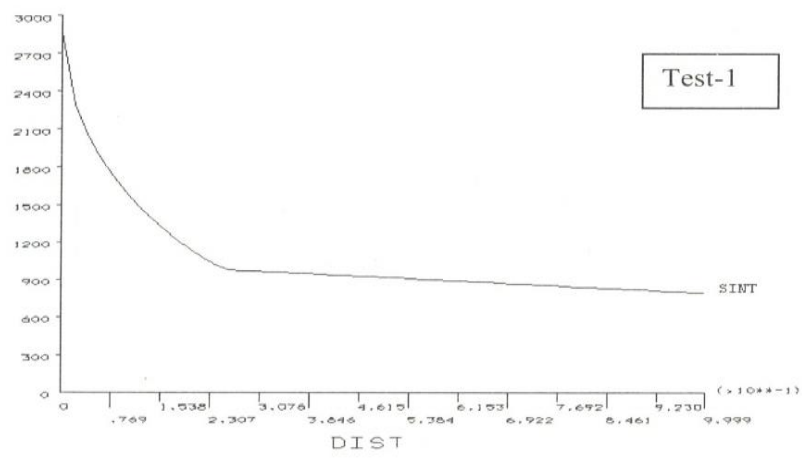

Fig. 3 Stress Intensity factor for the test conditions of width $=25 \mathrm{~mm}$,crack length $=2.5 \mathrm{~mm}$, load $=900 \mathrm{MPa}$

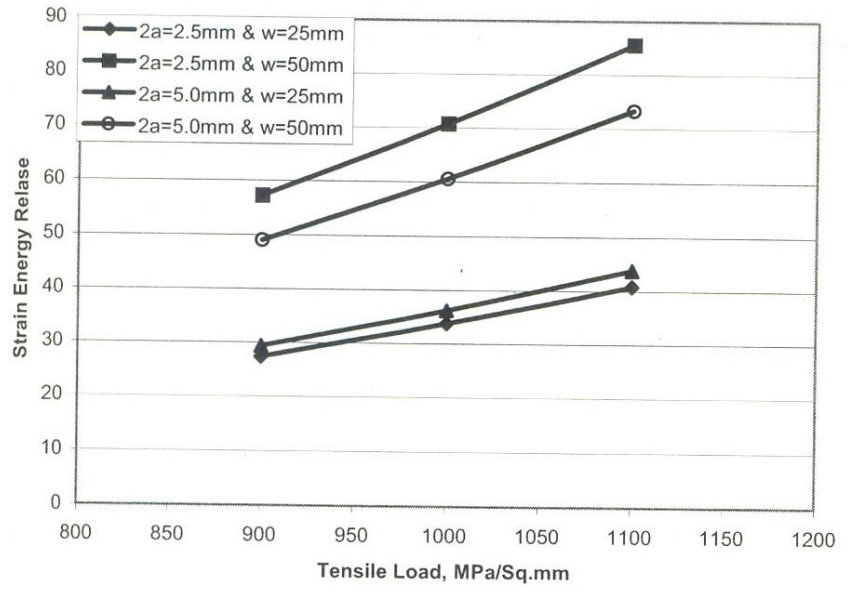

Fig. 4 Variation of strain energy with applied load

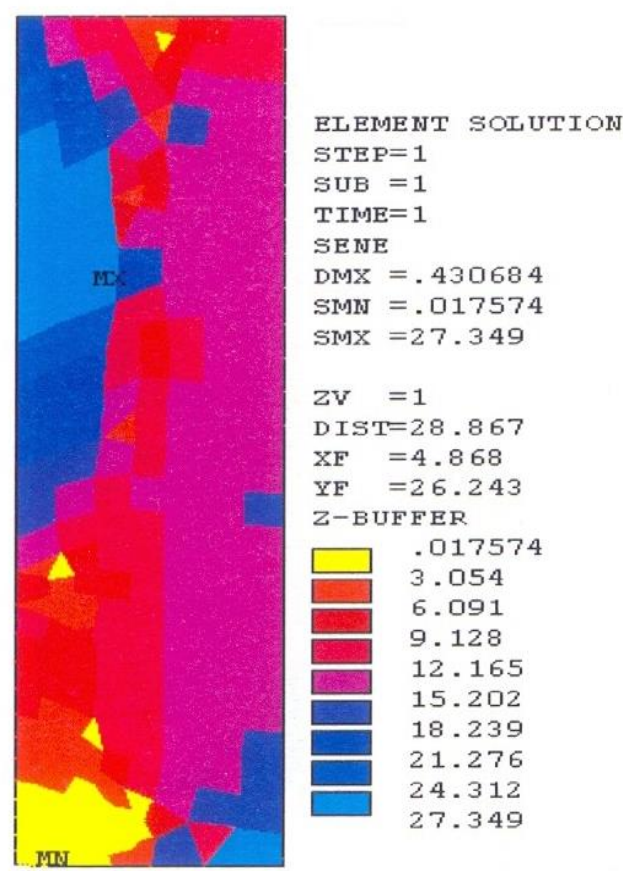

Fig. 5 Strain Release Energy for the test conditions of width $=25 \mathrm{~mm}$, crack length $=2.5 \mathrm{~mm}$, load $=900 \mathrm{MPa}$

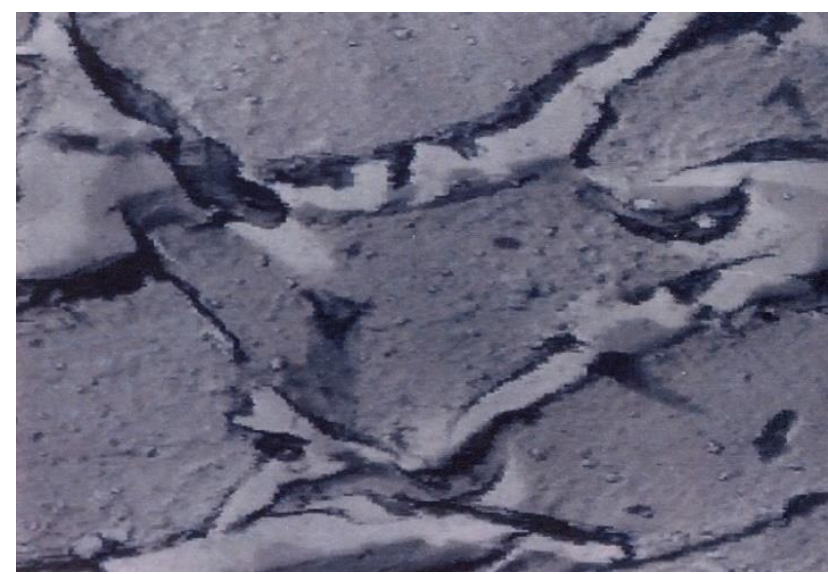

Fig. 6 Microstructure of fractured specimen for the test conditions of width $=25 \mathrm{~mm}$, crack length $=2.5 \mathrm{~mm}$, load $=900 \mathrm{MPa}$ 


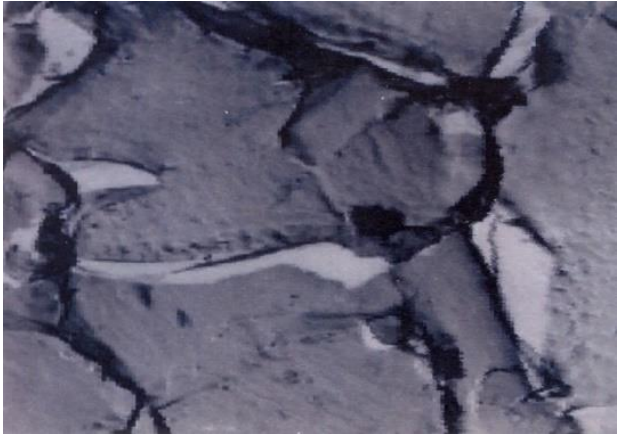

Fig. 7 Microstructure of fractured specimen for the test conditions of width $=50 \mathrm{~mm}$, crack length $=5 \mathrm{~mm}$, load $=900 \mathrm{MPa}$

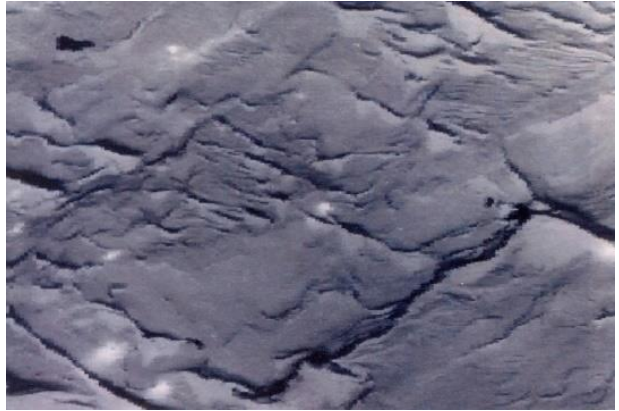

Fig. 8 Microstructure of fractured specimen for the test Conditions of width $=25 \mathrm{~mm}$, crack length $=2.5 \mathrm{~mm}$, load $=1000 \mathrm{MPa}$

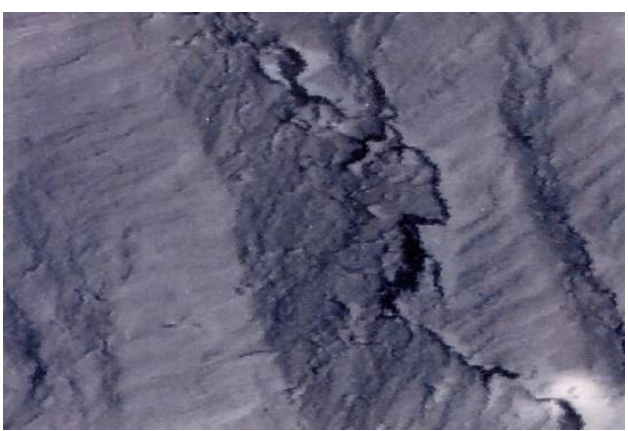

Fig. 9 Microstructure of fractured specimen for the test conditions of width $=50 \mathrm{~mm}$ crack length $=5 \mathrm{~mm}$, load $=1000 \mathrm{MPa}$

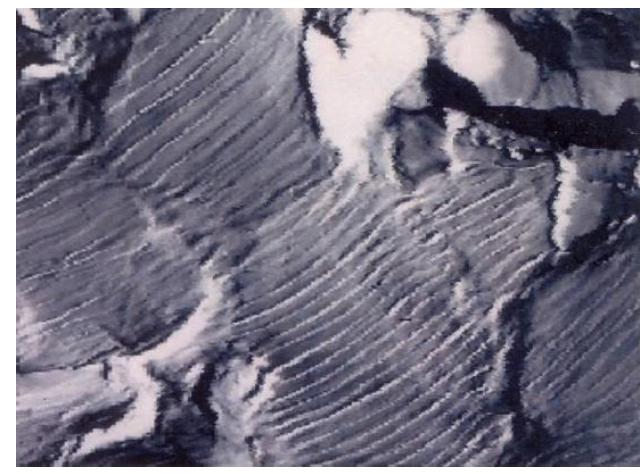

Fig. 10 Microstructure of fractured specimen for the test conditions of width $=25 \mathrm{~mm}$, crack length $=2.5 \mathrm{~mm}$, load $=1100 \mathrm{MPa}$

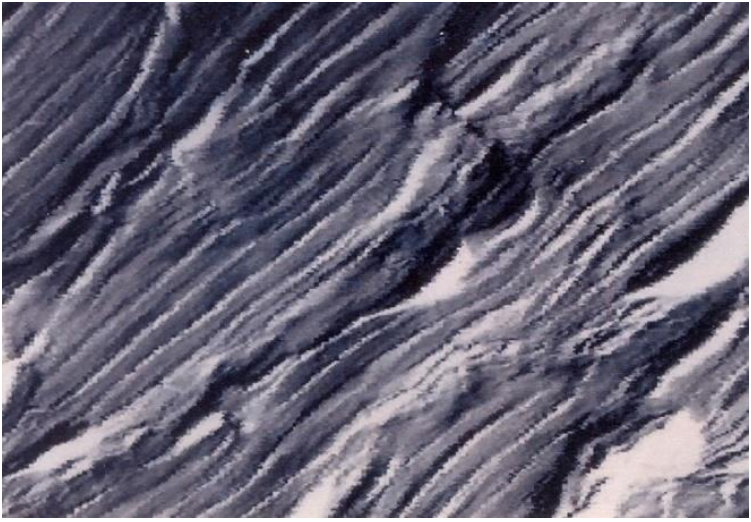

Fig. 11 Microstructure of fractured specimen for the test conditions of width $=50 \mathrm{~mm}$, crack length $=5 \mathrm{~mm}$, load $=1100 \mathrm{MPa}$

\section{Conclusions}

In this work the influence of crack size, specimen dimensions and tensile load on the Ti alloy was studied. The results obtained from the finite element analysis were compared with the experimental results. The following points are concluded from the present work:

1) Fracture-resistant design has two significant components. The first, which considers the applied stress state and likelihood of flaws being present in the structure, allows for estimating stresses in terms of flaw size and the material property, $\mathrm{K}_{\mathrm{I}}$.

2) There is a general trend of increasing stress intensity factor with increasing applied tensile load. It can also be observed that the stress intensity factor increases with increasing crack size and width of the flat specimen.

3) It is also revealed that the specimen having width $=50 \mathrm{~mm}$ and crack size of $5 \mathrm{~mm}$ exhibits steep rate of increasing stress intensity factor. The graph curvature is a polynomial of degree three.

4) Under all the test conditions, the strain energy release increases with increasing applied tensile load. Crack extension can occur when crackdriving force is equal to the energy required for crack growth. For a particular stress the energy release rate is proportional to the crack size. As the tensile load and the size of the initial increase, there is an increased crack growth in the Ti alloy.

5) In the examined Ti alloy under tension the cracks were observed to be initiated at the interface between $\alpha$ and $\beta$ phases and in $\alpha$ - grains for the specimens tested under $\sigma=900 \mathrm{MPa}$ and crack sizes of $25 \mathrm{~mm}$ and $50 \mathrm{~mm}$ respectively.

\section{References}

Sergio Baragetti(2014), Notch corrosion fatigue behavior of Ti-6Al-4V, Materials,7,4349-4366.

Kuntimaddi Sadananda, S. Sarkar, Daniel Kujawski, A.K. Vasudevan (2009), A two parameter analysis of S-N fatigue life using stress range and maximum stress, International Journal of fatigue , 31, 1648-1659. 
Yang Cao, Chunhuan Guo, Shifan Zhu, Ningxia Wei, Raja Ahsan Javed and Fengchun Jiang (2015), Fracture behavior of $\mathrm{Ti} / \mathrm{Al}_{3} \mathrm{Ti}$ metal-intermetaalic laminate (MIL) composite under dynamic loading, Materials Science and Engineering A, 637,235-242.

Chenchong Wang, Chi Zhang, Zhigang Yang, Jie Su, Yuging Weng (2015) , Analysis of fracture toughness in high Co-Ni secondary hardening steel using FEM, Materials Science and Engineering A,646,1-7.

Galal H. Senussi, Katarina Colic, Aleksandar, Sedmark (2011), Experimental analysis of fatigue fracture behavior of Ti6Al-4V alloys.

Burghard.H.C and Stoloff.N.S, Cleavage phenomena and topographic features, ASTM STP 436, 298-302.
Filice.L, F. Gagliardi, S. Lazzaro, C. Rocco (2010), FE simulation and experimental considerations on Ti alloy super plastic forming for aerospace applications, International Journal of Metal Forming, 3,141-46.

Abhay K.Jha, Satish KumarSingh, M.Swathi Kiranmayee, K.Sreekumar, P.P.Sinha, (2010), Failure Analysis of Titanium alloy (Ti-6Al-4V) fastener used in aerospace application, Engineering Failure Analysis, 17(6), $1457-$ 1465.

Shanyavsky.A.A, Stepanov.N.V, (1995) Fractographic Analysis of Fatigue Crack Growth in engine compressor disks of Ti$6 \mathrm{Al}-3 \mathrm{Mo}-2 \mathrm{Cr}$ titanium alloy, Fatigue \& Fracture of Engineering Materials \& Structures, 18,539-550.

Shanyavsky.A.A, Losev.A.I, Banovf.M.D, (1998) Development of fatigue cracking in aircraft engine compressor disks of Ti-6Al-3Mo-2Cr titanium alloy, Fatigue \& Fracture of Engineering Materials \& Structures, 21, 297-313. 